\title{
Nuclear localization signal in TRIM22 is essential for inhibition of type 2 porcine reproductive and respiratory syndrome virus replication in MARC-145 cells
}

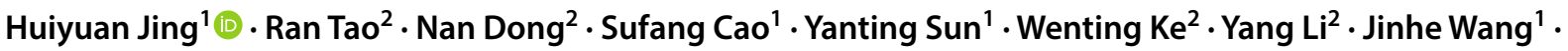 \\ Yan Zhang ${ }^{1} \cdot$ Hui Huang ${ }^{1} \cdot$ Wang Dong $^{1}$
}

Received: 19 February 2019 / Accepted: 23 July 2019 / Published online: 2 August 2019

๑) Springer Science+Business Media, LLC, part of Springer Nature 2019

\begin{abstract}
Porcine reproductive and respiratory syndrome virus (PRRSV) infection causes one of the most economically important swine diseases worldwide. Tripartite motif-containing 22 (TRIM22), a TRIM family protein, has been identified as a crucial restriction factor that inhibits a group of human viruses. Currently, the role of cellular TRIM22 in PRRSV infection remains unclear. In the present study, we analyzed the effect of TRIM22 on PRRSV replication in vitro and explored the underlying mechanism. Ectopic expression of TRIM22 impaired the viral replication, while TRIM22-RNAi favored the replication of PRRSV in MARC-145 cells. Additionally, we observed that TRIM22 deletion SPRY domain or Nuclear localization signal (NLS) losses the ability to inhibit PRRSV replication. Finally, Co-IP analysis identified that TRIM22 interacts with PRRSV nucleocapsid (N) protein through the SPRY domain, while the NLS2 motif of N protein is involved in interaction with TRIM22. Although the concentration of PRRSV N protein was not altered in the presence of TRIM22, the abundance of $\mathrm{N}$ proteins from simian hemorrhagic fever virus (SHFV), equine arteritis virus (EAV), and murine lactate dehydrogenaseelevating virus (LDV) diminished considerably with increasing TRIM22 expression. Together, our findings uncover a previously unrecognized role for TRIM22 and extend the antiviral effects of TRIM22 to arteriviruses.
\end{abstract}

Keywords Porcine reproductive and respiratory syndrome virus (PRRSV) - Nucleocapsid (N) protein - Tripartite motifcontaining 22 (TRIM22) · PRRSV-host interaction · Replication

\section{Introduction}

Classified within the order Nidovirales, family Arteriviridae, porcine reproductive and respiratory syndrome virus (PRRSV) causes reproductive failures in sows and respiratory diseases in growing pigs, leading to enormous economic loss to the pork industry worldwide [1]. Current

Edited by Juergen A Richt.

Huiyuan Jing

lhsjhy@126.com

1 Key Laboratory of Veterinary Biological Products, College of Veterinary Medicine, Henan University of Animal Husbandry and Economy, Long-zi-hu Street, Zhengzhou 450046, Henan, China

2 State Key Laboratory of Agricultural Microbiology, College of Veterinary Medicine, Huazhong Agricultural University, Wuhan 430070, China antiviral/vaccination strategies provide only limited protection from infections [2-5], due to suppression of the immune response by this virus [6-12]. The single-stranded positive sense RNA genome of this virus is approximately $15 \mathrm{~kb}$ in size and encodes at least 12 overlapping open reading frames (ORFs), including envelope glycoprotein, unglycosylated membrane protein, and nucleocapsid $(\mathrm{N})$ protein [13-15].

As the most abundant protein of PRRSV, $\mathrm{N}$ protein has been recognized to be involved in both viral replication and pathogenesis $[16,17]$. Besides encapsidation of viral RNA, $\mathrm{N}$ protein can also interact with nonstructural protein 2 (nsp2), nsp9, and N protein itself [18-20]. Most importantly, interplay between $\mathrm{N}$ protein and the host cell has been shown to modulate interferon (IFN), interleukin-10 (IL-10), IL-15 and SCD83 production and Tregs proliferation through activation of p38 MAPK (mitogen-activated protein kinases), NF- $\kappa B$ (Nuclear factor- $\kappa B$ ) and Sp1 signal pathways, thus facilitating viral infection [21-27]. Given the suppression of innate immunity by $\mathrm{N}$ protein, it is essential to identify host 
restriction factors and intrinsic immunity against PRRSV infection.

The tripartite motif (TRIM) family of proteins are defined by the highly conserved N-terminal RBCC (RING-finger B-Box Coiled-coil) signature domain [28, 29]. The RINGfinger domain is associated with E3 ubiquitin ligase activity, whereas the B-Box and Coiled-coil region is crucial for homo- and hetero-oligomerization [28, 29]. Functionally, TRIM proteins are implicated in a broad array of cellular processes that ranging from apoptosis, transcription, differentiation, and autophagy to, more recently antiviral immunity [30-32]. A prime example is TRIM22 (also referred to as Staf50), which has previously been identified as a target gene for tumor suppressor p53 and involved in cellular proliferation [33]. As a member of TRIM family, the RINGfinger domain was shown to be required for regulation of NF-кB [34, 35], apoptosis [36], NOD2 signaling [37], and autophagy-dependent Mycobacterium tuberculosis clearance [38]. However, TRIM22 also contains C-terminal SPRY domain to dictate the different subcellular distributions and specific functions which has not been fully clarified $[39,40]$.

Recently, the role of TRIM22 acting as a restriction factor to potentiate the intrinsic immunity has become increasingly clear [41]. Indeed, a broad antiviral spectrum has been described for TRIM22, including human immunodeficiency virus type 1 (HIV-1) [42, 43], DNA virus from the Hepadnaviridae family (hepatitis B virus, HBV) [44], positive-strand RNA viruses, including members of the family Picornaviridae (encephalomyocarditis virus, ECMV) [45], Flaviviridae (hepatitis C virus, HCV) [46], as well as negative-strand RNA viruses in the family Orthomyxoviridae (influenza A virus, IAV) [47]. Thus, the aim of present study is to seek evidence for a potential restrictive role for cellular TRIM22 in PRRSV infection.

\section{Materials and methods}

\section{Cells, viruses, and antibodies}

HEK293T and MARC-145 cells were cultured and maintained in Dulbecco's modified Eagle medium (DMEM) supplemented with $10 \%$ heat-inactivated fetal bovine serum (FBS) and then incubated at $37{ }^{\circ} \mathrm{C}$ in a humidified $5 \% \mathrm{CO}_{2}$ incubator. PRRSV strain HN1 (GenBank: AY457635.1) is a highly pathogenic type 2 (North American) PRRSV, which was isolated from the lung of pigs suffering from "high fever" syndrome in 2009 in Henan Province, China [48]. The virus was amplified and titered in MARC-145 cells.

Mouse monoclonal anti-hemagglutinin (HA), anti-Flag, and anti-actin antibodies were purchased from ABclonal Biotechnology. Anti-Flag, anti-HA polyclonal antibody (Macgene), TRIM22 polyclonal antibody (Abcam) were purchased and used according to the manufacturers' recommendations. HRP-conjugated anti-mouse and anti-rabbit IgG light (or heavy) chain specific antibodies (Abbkine Science, USA) were purchased and used according to the manufacturers' recommendations. A monoclonal antibody directed against PRRSV N protein was produced from hybridoma cells derived from $\mathrm{Sp} 2 / 0$ myeloma cells and spleen cells of $\mathrm{BALB} / \mathrm{c}$ mice immunized with recombinant $\mathrm{N}$ protein [49]. HRP-conjugated anti-mouse and anti-rabbit $\operatorname{IgG}$ antibodies were purchased from the Beyotime Institute of Biotechnology (Jiangsu, China).

\section{Plasmids constructions and mutagenesis}

Expression plasmids for human TRIM22 (NM_006074) were purchased from Vigene Bioscience and were cloned into the pCMV-Tag2B vector (Stratagene). The HA epitope tag was amplified by PCR and cloned into the pCAGGS-MCS vector to generate the pCAGGS-HA plasmid, encoding an N-terminal HA tag. To construct pCAGGS-HA-N, the cDNA fragment encoding full-length PRRSV N protein was amplified by PCR and inserted into the pCAGGS-HA plasmid [49]. Plasmids encoding truncated human TRIM22 or $\mathrm{N}$ protein muants were constructed by overlapping PCR amplification using the specific primers listed in Table $1 . \mathrm{N}$ protein expression plasmids for simian hemorrhagic fever virus (pCAGGS-HA-N/SHFV, NC_003092.2), equine arteritis virus (pCAGGS-HA-N/EAV, NC_002532.2), and lactate dehydrogenase-elevating virus (pCAGGS-HA-N/LDV, NC_001639.1) were purchased from TsingKe Biotechnology. All constructs were confirmed with DNA sequencing.

\section{Transfection and TRIM22 gene silencing by siRNA}

The HEK293T cells were transiently transfected with expression plasmids using Lipofectamine 2000 (Invitrogen) according to the manufacturer's instructions. MARC145 cells were transfected with expression plasmids using Lipofectamine 3000 . Where necessary, empty control plasmid was added to ensure that each transfection receives the same amount of total DNA. For siRNA knockdowns, cells plated in 6-well plates were transfected with $30 \mathrm{nM}$ the indicated siRNAs twice over a $48 \mathrm{~h}$ period by using Lipofectamine 2000. SiRNA sequences targeting monkey TRIM22 (NM_001113359.1) used are as follows: siTRIM22, 5'-CACCAAACAUUCCGCAUAATT-3'; Negative control-siRNA, 5'-UUCUCCGAACGUGUCACG UTT-3'. The cells were infected with PRRSV 24-36 h after transfection when indicated and incubated for the indicated times. Whole-cell lysates were then prepared and subjected to Western blotting assays. 
Table 1 The sequences of primers used for construction of TRIM22 (GenBank: NM_006074), and N (GenBank: KY964305.1) protein mutants

\begin{tabular}{|c|c|}
\hline Name & Primer sequence $\left(5^{\prime}-3^{\prime}\right)$ \\
\hline TRIM22-F & TTTAAGCTTATGGATTTCTCAGTAAAGGTAGACATA \\
\hline TRIM22-R & TTTCTCGAGGGAGCTCGGTGGGCACACAGTCATGGG \\
\hline$\Delta$ RING-F & TTTAAGCTTCAGACCAGATTCCAGCCTGGGAACCTC \\
\hline SPRY-F & TTTAAGCTTAAGAAGCCAAAATCTGTTTCCAAGAAA \\
\hline$\Delta$ SPRY-R & TTTCTCGAGGTACTGGACATCTGTCAGCTCTTTAAG \\
\hline$\Delta \mathrm{NLS}-\mathrm{F}$ & GAGTGAAAGCTGGACATTGAGTGTATTCCGAGTACCAG \\
\hline$\Delta \mathrm{NLS}-\mathrm{R}$ & CTGGTACTCGGAATACACTCAATGTCCAGCTTTCACTC \\
\hline ORF7-F & TTT $\underline{G A A T T C A T G C C A A A T A A C A A C G G C A A G C A G C}$ \\
\hline ORF7-R & TTTAGATCTTCATGTTGGGGGTGATGCTGTGGCG \\
\hline K10-4A-F1 & AGCAAGCGGCAGCGGCGGGGAATGGCCAGCCAGTCGA \\
\hline K10-4A-F2 & ATAACAACGGCAAGCAGCAAGCGGCAGCGGCGGGGAATG \\
\hline $\mathrm{C} 23 \mathrm{~A}-\mathrm{F} 1$ & AGCCAGTCGATCAGCTGGCCCAAATGCTGGGTAAGATCA \\
\hline C23A-F2 & AAAGAAAAAGAAGGGGAATGGCCAGCCAGTCGATCAGCTGG \\
\hline C23A-F3 & ATAACAACGGCAAGCAGCAAAAGAAAAAGAAGGGGAA \\
\hline Q32-4A-F & CATCGCCGCAGCAGCCGCGTCCAGAGGCAAGGGACCG \\
\hline Q32-4A-R & CTCTGGACGCGGCTGCTGCGGCGATGATCTTACCCAG \\
\hline $\mathrm{K} 43-2 \mathrm{~A}-\mathrm{F}$ & ACCGGGGGCGGCAAATAGGAAGAAAAACCCG \\
\hline K43-2A-R & CTTCCTATTTGCCGCCCCCGGTCCCTTGCCTCTG \\
\hline P50-3A-F & CGGAGAAGGCCCATTTCGCTCTAGCGACTGAAGATGACG \\
\hline P50-3A-R & GCGAAATGGGCCTTCTCCGCGTTTTTCTTCCTATTTTTC \\
\hline
\end{tabular}

The restriction enzyme sites used for cloning are underlined in italics. Locations of mutations are underlined

\section{TCID $_{50}$ assay for PRRSV}

PRRSV titers were expressed as the tissue culture infectious dose $50\left(\mathrm{TCID}_{50}\right)$ per milliliter using the Reed-Muench method as previous described [12]. Briefly, MARC-145 cells were seeded in 96-well plates, following, infected with serial 10-fold dilutions of PRRSV samples in eight replicates. Plates were incubated for $72-96 \mathrm{~h}$ before virus titers were calculated.

\section{Quantitative RT-PCR (qPCR)}

Total RNA was isolated from MARC-145 cells using TRIzol Reagent (Invitrogen). qPCR was performed with SYBR ${ }^{\circledR}$ Green Realtime PCR Master Mix (Toyobo Biologics, Japan) in a ABI 7500 system. The individual transcripts in each sample were assayed three times. The PCR conditions were as follows: initial denaturation for $10 \mathrm{~min}$ at $95^{\circ} \mathrm{C}$, followed by 40 cycles of $15 \mathrm{~s}$ at $95{ }^{\circ} \mathrm{C}, 15 \mathrm{~s}$ at $58^{\circ} \mathrm{C}$, and $40 \mathrm{~s}$ at $72{ }^{\circ} \mathrm{C}$. The fold change in gene expression relative to the normal control was calculated with the $\Delta \Delta \mathrm{CT}$ method. Primers (Table 2) were designed using the Primer Express software (version 3.0; Applied Biosystems, Carlsbad, CA). Absolute quantitative mRNA levels were calculated using standard curves as previously described [50].

\section{Western blotting analysis}

Cytoplasm and nuclear protein extracts from PRRSV-infected MARC-145 cells were prepared with the cytoplasmic and nuclear protein extraction kit (Aidlab Biotechnologies) according to the manufacturer's protocols. Cells cultured in $60-\mathrm{mm}$ dishes were prepared by adding $120 \mu \mathrm{L}$ of $2 \times$ lysis buffer A (65 mM Tris-HCl [pH 6.8], $4 \%$ sodium dodecyl sulfate (SDS), 3\% DL-dithiothreitol, and 40\% glycerol). The cell extracts were boiled for $10 \mathrm{~min}$, and then resolved with 8-12\% SDS-PAGE. The separated proteins were electroblotted onto a polyvinylidenedifluoride (PVDF) membrane (Millipore, Billerica, MA). Run for $1-2 \mathrm{~h}$ at $100 \mathrm{~V}$ on ice. The Western blotting was probed with specific antibodies. The expression of $\beta$-actin was detected with an mouse monoclonal antibody
Table 2 The sequences of primers used for real-time PCR

\begin{tabular}{lll}
\hline Name & Forward primer $\left(5^{\prime}-3^{\prime}\right)$ & Reverse primer(5'-3') \\
\hline TRIM22 (NM_001113359.1) & TCAGTGACCATCTCAAGAGG & CACAAACCCAGCAAATGAC \\
GAPDH (NM_001195426.1) & TCATGACCACAGTCCATGCC & GGATGACCTTGCCCACAGCC \\
Viral total RNA & AAACCAGTCCAGAGGCAA & CGGCAAACTAAACTCCACA \\
\hline
\end{tabular}


to demonstrate equal protein sample loading. Densitometry quantification of protein bands of interest was performed using ImageJ software.

\section{Indirect immunofluorescence assay (IFA)}

MARC-145 cells seeded on microscope coverslips and placed in 24-well dishes were infected with PRRSV $(\mathrm{MOI}=0.5)$. At $24 \mathrm{hpi}$, the cells were fixed with $4 \%$ paraformaldehyde for $10 \mathrm{~min}$, and then permeated with $0.1 \%$ Triton X-100 for $10 \mathrm{~min}$ at room temperature. After three washes with PBS, the cells were sealed with PBS containing $5 \%$ bovine serum albumin for $1 \mathrm{~h}$, and then incubated separately with rabbit polyclonal antibody directed against TRIM22 (1:200) and mouse monoclonal antibody directed against PRRSV N protein (1:200) for $1 \mathrm{~h}$ at $37^{\circ} \mathrm{C}$. The cells were then treated with fluorescein-isothiocyanate-labeled goat anti-rabbit or Cy3-labeled goat anti-mouse antibodies (Invitrogen) for $1 \mathrm{~h}$, followed by 4',6-diamidino-2-phenylindole (DAPI) for $10 \mathrm{~min}$ at room temperature. After the samples were washed with PBS, fluorescent images were acquired with a confocal laser scanning microscope (Fluoview ver. 3.1; Olympus, Tokyo, Japan).

\section{Co-immunoprecipitation (Co-IP) and immunoblotting analyses}

To investigate the interactions between proteins, HEK293T or MARC-145 cells were lysed in immunoprecipitation lysis buffer (RIPA). After the lysates were incubated for $1 \mathrm{~h}$ at $37{ }^{\circ} \mathrm{C}$ with DNase $(100 \mu \mathrm{g} / \mathrm{mL})$ and RNase A $(100 \mu \mathrm{g} / \mathrm{mL})$, the lysate proteins were incubated overnight at $4{ }^{\circ} \mathrm{C}$ with the indicated antibodies. Protein $\mathrm{A}+\mathrm{G}$ agarose beads ( 30 $\mu \mathrm{L}$; Beyotime) were then added to each immunoprecipitation reaction for another $6 \mathrm{~h}$. The agarose beads were then washed three times and the captured proteins resolved on

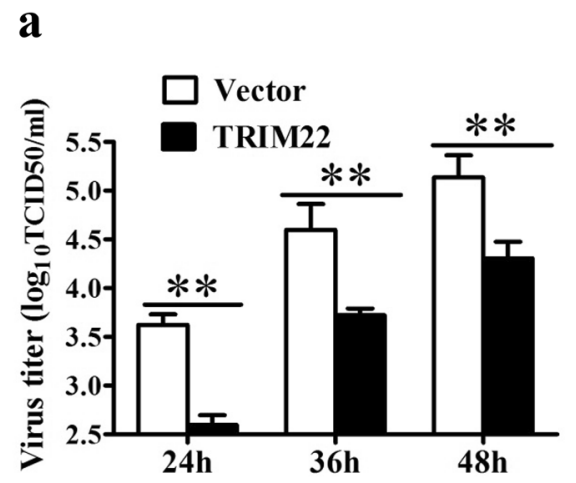

Fig. 1 Ectopic of TRIM22 impairs PRRSV replication in MARC145 cells. MARC-145 cells transfected with plasmid expressing Flag-tagged TRIM22 or an empty vector control were infected with PRRSV at an MOI of 0.5. a Progeny virus production were analyzed
8-12\% SDS-PAGE, transferred to PVDF membrane, and analyzed with immunoblotting.

\section{Statistical analysis}

The results represent the means and standard deviations from three independent experiments. GraphPad Prism 5 software (GraphPad Software, San Diego, CA, USA) was used for data analysis using a two-tailed unpaired $t$ test. Differences between groups were considered statistically significant when the $P$ value was less than $0.05 \quad(* P=0.05$; $* * P=0.01)$.

\section{Results}

\section{Ectopic expression of TRIM22 inhibited propagation of PRRSV}

Accumulating data suggest that TRIM22 is a restriction factor of several human viral infection [41]. However, whether TRIM22 participates in host defense against PRRSV is largely unknown. We first examined the impact of TRIM22 over-expression on PRRSV infection. To this end, MARC145 cells grown in 24-well plates were transfected with the Flag-TRIM22 (2 $\mu \mathrm{g})$ and followed by infected with PRRSV strain HN1 at an multiplicity of infection (MOI) of 0.5 . Supernatants collected at the 24,36 , and 48 h postinfection (hpi) were analyzed for virus titers with $\mathrm{TCID}_{50}$. As shown in Fig. 1a, over-expression of TRIM22 resulted in a significantly decrease of virus titers in supernatants of PRRSV-infected MARC-145 cells at 24 and 36 hpi (Fig. 1a). This antiviral effect of TRIM22 persisted at later time points (48 hpi). To further corroborate this finding, the effect of TRIM22 over-expression on the expression of corresponding viral total RNA in PRRSV-infected MARC-145 cells were

b

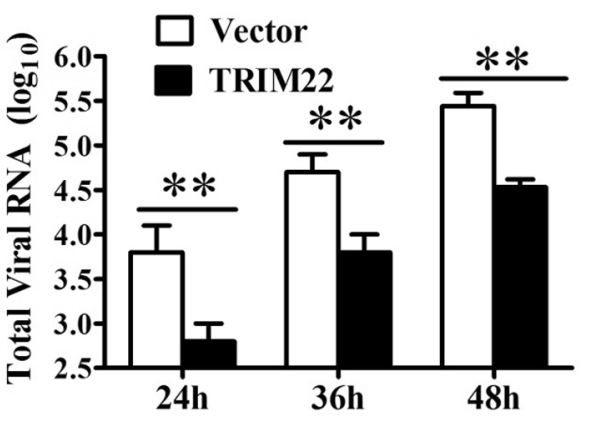

by $\mathrm{TCID}_{50}$ at the indicated time points post-infection. b The mRNA expression level of viral total RNA by qPCR were analyzed at the time points shown 
examined by qPCR. As expected, RNA qPCR results were in accordance with the TCID $_{50}$ data (Fig. 1b), suggesting that the over-expression of TRIM22 impairs the replication efficiency of PRRSV.

\section{Knockdown of TRIM22 augmented the replication of PRRSV}

Next, the effect of TRIM22 knockdown on PRRSV propagation was dissected by using siRNAs. To this end, MARC-145 cells were transfected with the siRNAs targeting TRIM22 and were then harvested for evaluating the knockdown efficiency of TRIM22 by real-time PCR and Western blotting analyses. As shown in Fig. 2a and $b$, compared with cells transfected with control siRNAs

$\mathbf{a}$

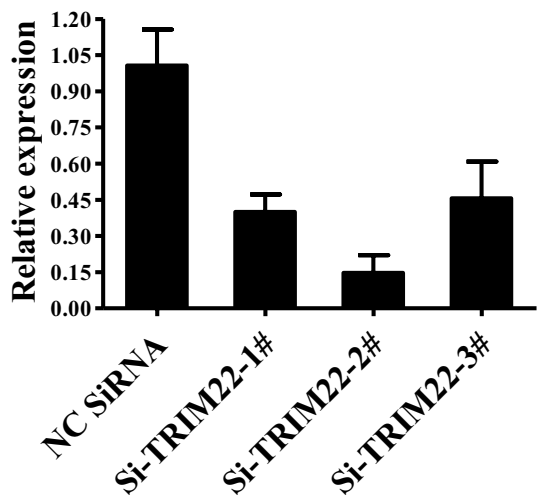

c

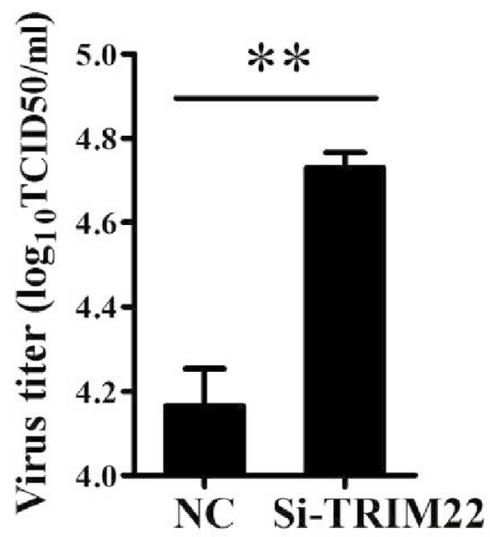

Fig. 2 Enhancement of PRRSV replication by TRIM22 knockdown. a TRIM22 mRNA level were examined by real-time PCR in negative control-siRNA (NC) or si-TRIM22 treated MARC-145 cells to confirm the knockdown efficiency of endogenous TRIM22. b MARC145 cells were transfected with siRNA targeting TRIM22 or NC siRNA. The cells were harvested at $36 \mathrm{~h}$ post-transfection and the silencing efficiency of TRIM22 was examined by Western blotting with an anti-TRIM22 antibody. The relative levels of TRIM22 were shown below the images after normalization with $\beta$-actin in densi-
(NC), the cells transfected with TRIM22-specific siRNAs exhibited a significantly decreased level $(\sim 85 \%)$ of TRIM22 expression (Fig. 2a, b).

To investigate the role of endogenous TRIM22 on PRRSV replication, MARC-145 cells were treated with control-siRNA or si-TRIM22 followed by PRRSV infection, then viral titers were measured by $\mathrm{TCID}_{50}$, and viral total RNA were quantified by qPCR at $36 \mathrm{hpi}$. Compared to control cells, we observed that there was a statistically significant increase of the virus yields in the supernatants of si-TRIM22-transfected cells (Fig. 2c). At the same time, a slight increase of PRRSV total RNA level were observed after silencing of TRIM22 (Fig. 2d), suggesting that the knockdown of endogenous TRIM22 favors the multiplication of PRRSV in MARC-145 cells.

b

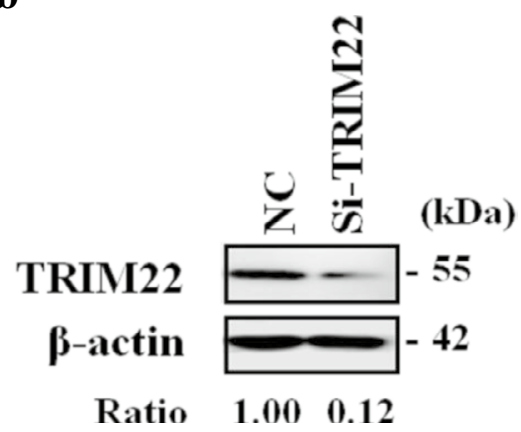

d

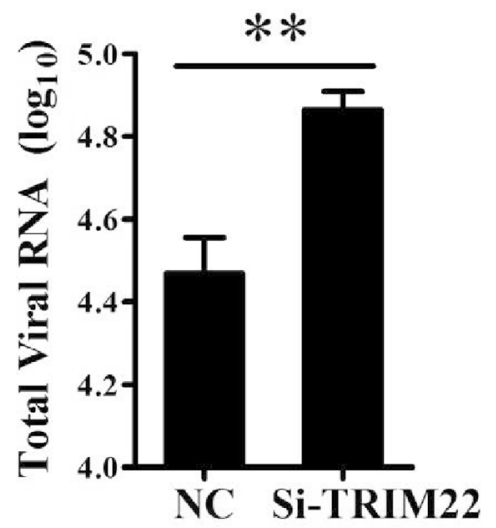

tometry analysis. c MARC-145 cells transfected with the si-TRIM22 or NC siRNA for $24 \mathrm{~h}$ were infected with PRRSV at MOI of 0.1 and the virus titers were examined at 36 hpi. d PRRSV RNA replication in TRIM22-silenced MARC-145 cells. MARC-145 cells transfected with the si-TRIM22 or NC siRNA for $24 \mathrm{~h}$ were infected with PRRSV at MOI of 0.1 and collected at $36 \mathrm{hpi}$. The total cellular RNA was extracted and the mRNA levels of PRRSV N gene were determined by qPCR 


\section{RING domain was not required for restriction of PRRSV}

In the case of ECMV, HBV, IAV, and HCV, the N-terminal RING domain of TRIM22 was invariably shown to be essential for viral restriction [44-47]. To investigate the exact antiviral mechanism of TRIM22 against PRRSV, three truncations of TRIM22 lacking only the RING domain, the RING, B-box and coiled-coil domains, or the SPRY domain were constructed and tested for repression activity (Fig. 3a). Unexpectedly, the results presented in Fig. 3b showed that the propagation of PRRSV was still restricted by ectopic expression of TRIM22 $\Delta$ RING. However, when challenged with PRRSV, an inhibitory effect on progeny virus production were observed in TRIM22 WT, and SPRY, except in $\triangle$ SPRY expression cells (Fig. 3b). Concordant with this, over-expression of TRIM22 SPRY domain led to a sharp decline in PRRSV total RNA expression compared with cells transfected with empty vector (Fig. 3c). Collectively, these data implied that TRIM22 exerts its antiviral function towards viruses of distinct families via distinct molecular determinants, and the SPRY domain is involved in PRRSV suppression.

\section{Nuclear localization signal of TRIM22 is essential for PRRSV inhibition}

The localization of TRIM22 has been associated with its antiviral function, as exemplified by TRIM22 restriction of HIV [51, 52]. To analysis the localization of TRIM22 after PRRSV infection, MARC-145 cells were infected with PRRSV, and confocal immunofluorescence assay were performed. As shown in Fig. 4a, endogenous TRIM22 localized in both the nucleus and the cytoplasm in MARC-145 cells. However, TRIM22 obviously redistributed from cytoplasm into the nucleus in PRRSV-infected cells, where they partially colocalized with $\mathrm{N}$ protein.

To further confirm the localization of TRIM22, the cytoplasmic and nuclear extracts of PRRSV-infected MARC-145 cells were prepared and endogenous TRIM22 was detected by Western blotting using anti-TRIM22 antibody. In parallel, Western blotting showed TRIM22 was upregulated in the nucleus, but was downregulated in the cytoplasm of PRRSV-infected MARC-145 cells. This indicated that PRRSV-induced translocation of TRIM22 into the nucleus.

Peptide sequence analysis of the TRIM22 protein using the PSORT program has identified two nuclear localization signal (NLS), a pat-4, 265-KKPK-268, and a pat-7 269-PVSKKLK-275 [53]. Given that the NLS was shown to be necessary for nuclear localization [39], these amino
Fig. 3 RING domain was not required for restriction of PRRSV. a Full-length and serial truncations of TRIM22 with deletion $(\Delta)$ of various domains. Numbers indicate the residues where deletions begin or end. MARC-145 cells transfected with Flag-TRIM22 (WT or domain lacking mutants) were infected with $0.5 \mathrm{MOI}$ of PRRSV for $48 \mathrm{~h}$. b Production of progeny virus were determined using TCID $_{50}$ assay. c The total cellular RNA was extracted and the mRNA levels of PRRSV N gene were determined by qPCR
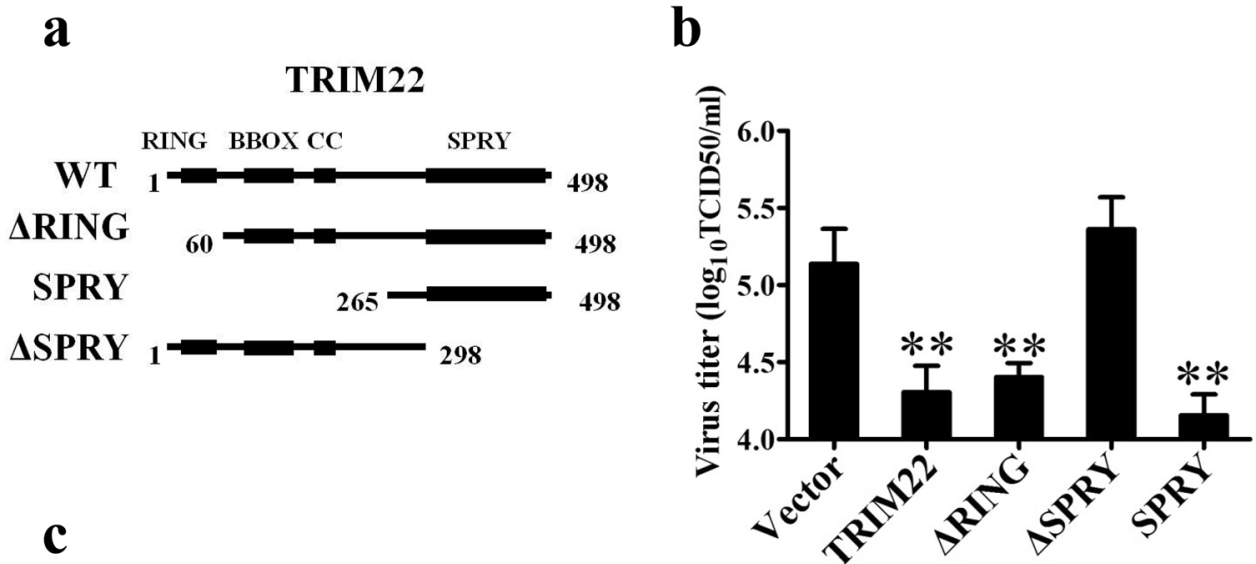
Fig. 4 Nuclear localization signal of TRIM22 is essential for PRRSV inhibition. a Subcellular distribution of TRIM22 after PRRSV infection in MARC-145 cells. MARC-145 cells were infected with PRRSV HN1 at a MOI of 1 , and were fixed for immunofluorescence analysis of TRIM22 (green), N protein (red) and nucleus marker DAPI (blue) localization. b Nuclear and cytoplasmic fractionation of MARC-145 cells infected with PRRSV for $36 \mathrm{~h}$. Each nuclear and cytosolic fraction was prepared and subjected to Western blotting analysis with an antibody specific for TRIM22, LaminA + C as a nuclear protein marker, HSP90 as a cytosolic protein marker, or the PRRSV N protein. $\mathbf{c}$ The position and sequence of the NLS in TRIM22. MARC-145 cells transfected with FlagTRIM22 (WT or NLS lacking mutants) were infected with $0.5 \mathrm{MOI}$ of PRRSV for $48 \mathrm{~h}$. d Production of progeny virus were determined using TCID $_{50}$ assay. e The total cellular RNA was extracted and the mRNA levels of PRRSV N gene were determined by qPCR (Color figure online) $\mathbf{a}$

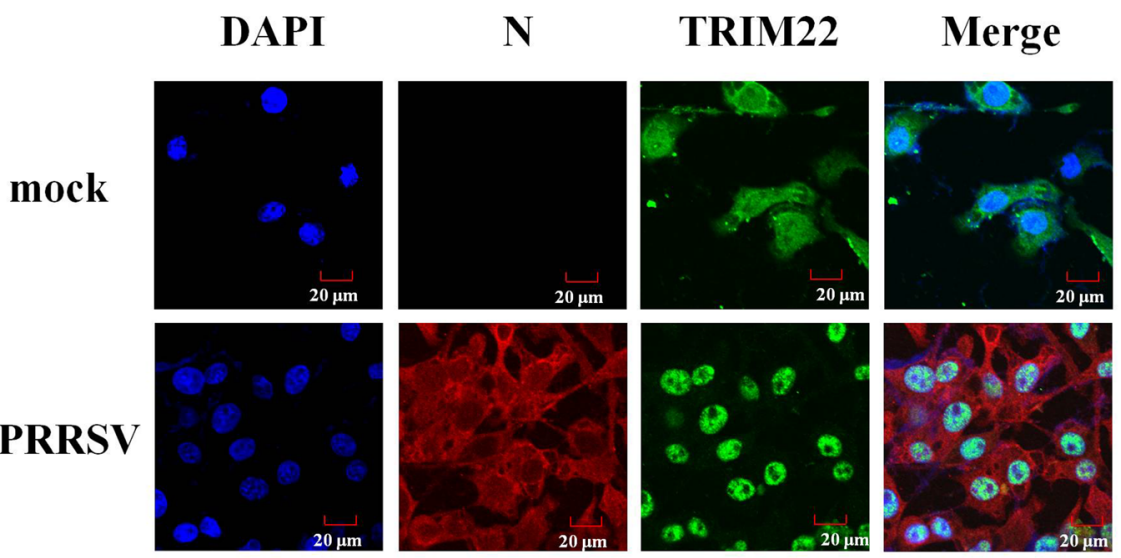

b

c

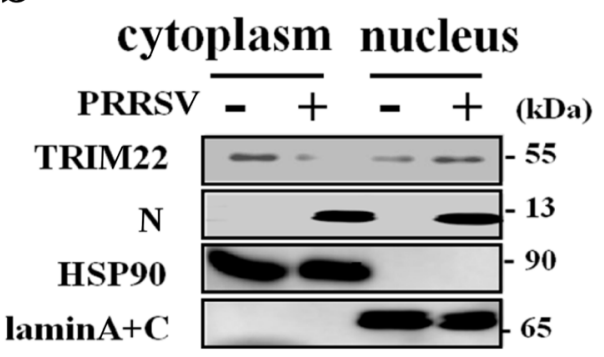

TRIM22

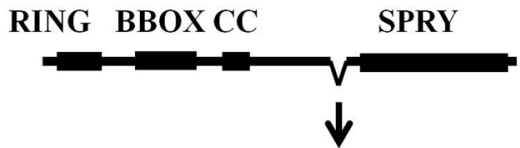

NLS 265-KKPKPVSKKLK-275

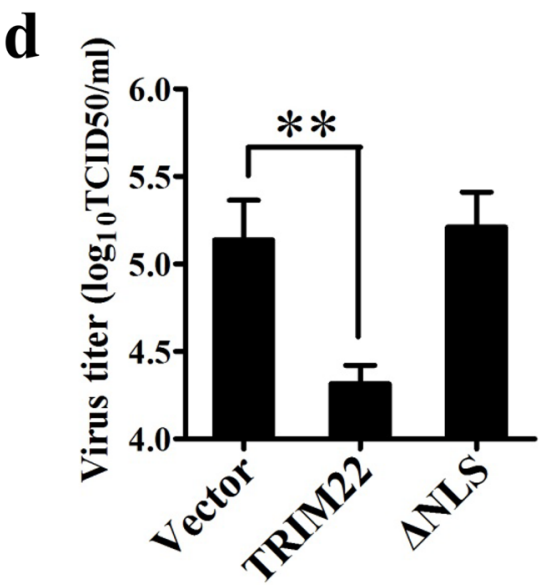

e

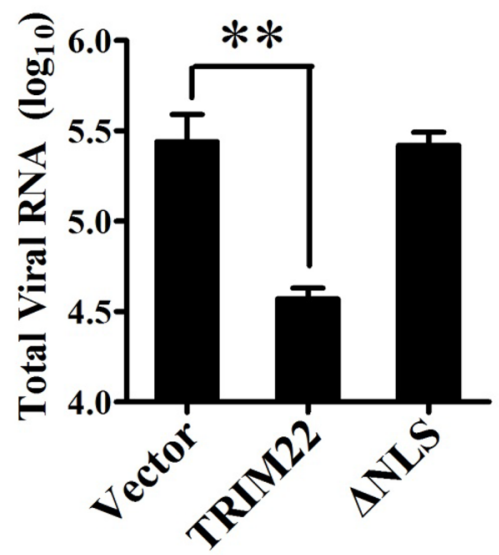

acids were deleted to give rise TRIM22 NLS mutant (Fig. $4 \mathrm{c}$ ). TCID $_{50}$ assay was then performed to determine whether the NLS of TRIM22 is related to PRRSV antagonism. As shown in Fig. 4d, the cells transfected with the wild-type Flag-TRIM22 expressing construct exhibited a significant reduction in virus production, as compared with cells transfected with the empty vector. However, the NLS deleted TRIM22 showed minimal or no effect on PRRSV replication. Consistent with the results of $\mathrm{TCID}_{50}$, the PRRSV total RNA level in transfected cells was significantly suppressed by TRIM 22 as determined by qPCR (Fig. 4e). This result directly suggested that the NLS is important for TRIM22 to curtail the replicative activity of PRRSV.

\section{TRIM22 interacted with the N protein of PRRSV}

Since TRIM22 exerts its antiviral function in the nucleus, we then tested whether TRIM22 might interact with nuclearlocalized proteins encoded by PRRSV, such as the nucleocapsid protein. To this end, HEK293T cells were transfected with Flag-TRIM22-expressing plasmid and HA-N-expressing plasmid individually or together. The cell lysates were immunoprecipitated with an anti-HA mAb 
and were then subjected to Western blotting. It was shown that $\mathrm{N}$ protein could be precipitated in the cells that were co-transfected with the plasmids pCAGGS-HA-N and pCMV-Flag-TRIM22 (Fig. 5a). The reciprocal IP assay was also performed and the same results were observed (Fig. 5a). We also tested whether TRIM22 could interact with three PRRSV proteins: Nsp1 $\alpha$, Nsp1 $\beta$, and Nsp11, but no interaction were detected by Co-IP (data not shown).

We further examined the interaction of endogenous TRIM22 with the $\mathrm{N}$ protein in the context of PRRSV infection. MARC- 145 cells were infected with PRRSV for $30 \mathrm{~h}$ before harvested and immunoprecipitated with an antiTRIM22 mAb. As shown in Fig. 5b, by Co-IP assay suggested that endogenous TRIM22 could interact with the $\mathrm{N}$ protein of PRRSV in MARC-145 cells.

\section{The regions responsible for the interaction of TRIM22 with $\mathrm{N}$ protein}

Several functional motif in $\mathrm{N}$ protein has been reported to interacts with the host cellular proteins, including NLS1, NLS2, N-N interaction motif and an SH3 binding motif (Fig. 6a) [54-57]. To determine the regions of $\mathrm{N}$ protein responsible for the interaction with TRIM22, a serial of truncated mutants, as indicated, were co-transfected with pCMV-Flag-TRIM22 in HEK293T cells. The cell lysates were precipitated with an anti-HA or anti-Flag mAb. As shown Fig. 6b, TRIM22 was strongly co-precipitated with HA-N, K10-4A, C23A, Q32-4A, and P50-3A. No coprecipitation was detectable with extracts from $\mathrm{K} 43-2 \mathrm{~A}$ expressing cells or cells tranfected with the empty vector, indicating that the NLS2 of N protein is involved in interaction with TRIM22.

We next identified the domain of TRIM22 that is essential for the interaction between TRIM22 and N protein. Four truncations of TRIM22 lacking only the RING domain, the
RING, B-box and coiled-coil domains, the SPRY domain, or the NLS, were constructed to identify the domains required for the interaction between TRIM22 and N protein. The results showed that the truncated TRIM22 which lack the RING domain or B-box and coiled-coil domains retained the ability to interact with $\mathrm{N}$ protein, while TRIM22 mutants which lack the SPRY domain or NLS were unable to interact with N protein, indicating that the SPRY domain and NLS are important for the interaction between TRIM22 and $\mathrm{N}$ protein (Fig. 6c).

\section{TRIM22 destabilizes the N proteins from the SHFV, EAV, and LDV}

Finally, we examined whether TRIM22-N protein interaction could be expanded to other members in family Arteriviridae, such as simian hemorrhagic fever virus (SHFV), equine arteritis virus (EAV), and murine lactate dehydrogenase-elevating virus (LDV). For this purpose, HEK293T cells were co-transfected with Flag-tagged TRIM22-expressing plasmid and HA-tagged $\mathrm{N}$ protein-expressing plasmids, Co-IP experiments were then performed to assess possible interactions. Unexpectedly, the N protein levels of SHFV (Fig. 7a), EAV (Fig. 7b), and LDV (Fig. 7c) decreased sharply upon co-transfection with TRIM22. As shown Fig. 7a-c, evident down-regulation of SHFV, EAV and LDV N protein could be detected, indicating TRIM22 targets arteriviruses N protein in a species-specific manner.

\section{Discussion}

To date, the most intensively studied antiviral TRIM protein may be TRIM5 $\alpha$, especially to retrovirus [58]. Being one of its closest paralogs, TRIM22 was first identified independently by several laboratories to possess antiviral
Fig. 5 Interaction of the $\mathrm{N}$ protein with TRIM22. a HEK293T cells were co-transfected with Flag-TRIM22 and HA-N. The cell lysates were immunoprecipitated with an anti-HA (or Flag) antibody and detected with an anti-HA or anti-Flag antibody. Whole-cell lysates (WCL) were probed with Flag and HA antibody. $\mathbf{b}$ The interaction of $\mathrm{N}$ protein with endogenous TRIM22. MARC-145 cells were infected with PRRSV. The cell lysates were immunoprecipitated with an anti-TRIM22 antibody and detected with an anti-N protein antibody

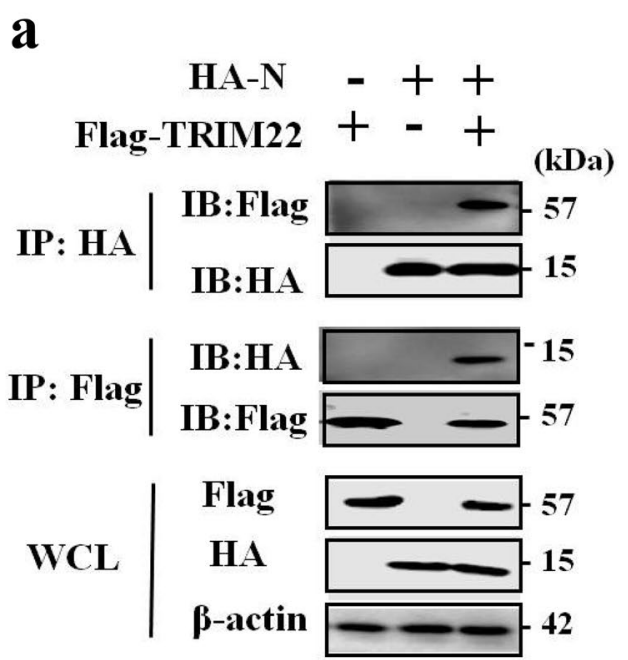

b

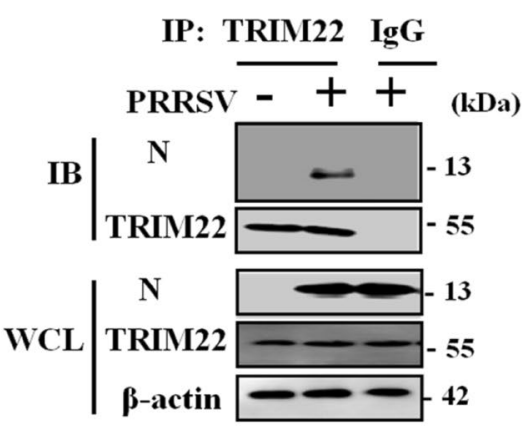


Fig. 6 The regions responsible for the interaction of TRIM22 with $\mathrm{N}$ protein. a The schematic diagram of $\mathrm{N}$ protein individual mutants investigated in this study. b The interaction of TRIM22 with $\mathrm{N}$ protein mutants by Co-IP. HEK293T cells were co-transfected with the indicated plasmids. The cell lysates were immunoprecipitated with an anti-HA mAb and further probed with an anti-HA or anti-Flag antibody. $\mathbf{c}$ The interaction of $\mathrm{N}$ protein with TRIM22 deletion mutants by Co-IP. HEK293T cells were cotransfected with the indicated plasmids. The cell lysates were immunoprecipitated with an anti-Flag $\mathrm{mAb}$ and further probed with an anti-HA or antiFlag polyclonal antibody

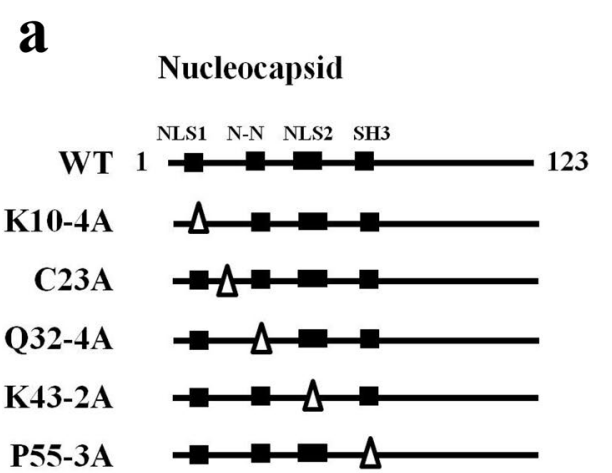

b

b

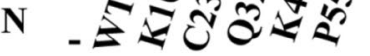

Flag-TRIM22 - $\overline{++++++}$ (kDa)

IP: |IB:Flag $\longrightarrow--\square$

HA IB:HA $\longrightarrow-15$

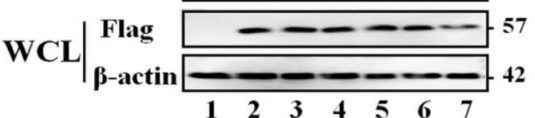

C

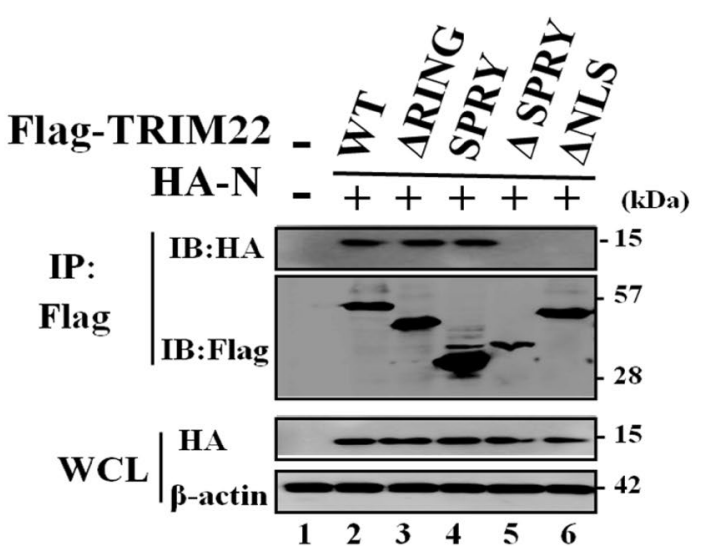

Fig. 7 TRIM22 reduces the expression levels of SHFV, EAV and LDV N proteins. HEK293T cells were cotransfected with arteriviruses $\mathrm{N}$ protein-expressing plasmids $(0.5 \mu \mathrm{g})$ and TRIM22-expressing plasmid as indicated. The SHFV (a), EAV (b) and LDV (c). $\mathrm{N}$ proteins expression were analyzed by Western blotting $30 \mathrm{~h}$ later in whole-cell lysates

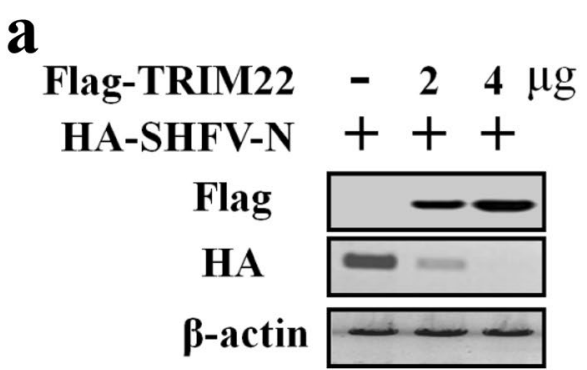

b

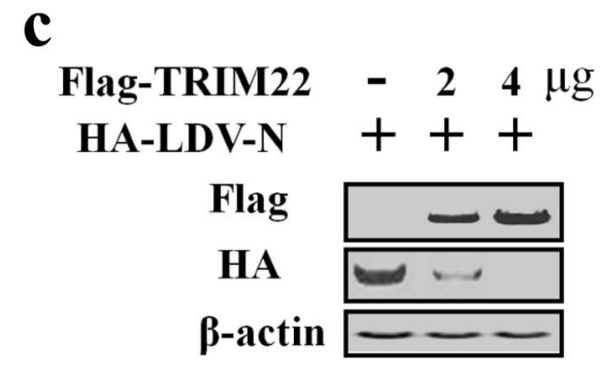

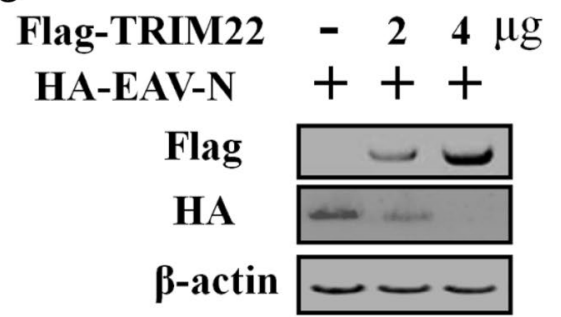

activity, targeting HIV-1 in particular [42, 43, 52, 53]. The RING domain of several TRIM proteins has been shown to possess an E3 ubiquitin ligase activity [41], either by targeting specific viral components (protein) for degradation or through governing the ubiquitination state of cellular proteins in innate immune signaling [34, 35, 37, 59-61].
Recently published data support this notion, showing that TRIM22 restricts EMCV, IAV, and HCV by degrading viral $3 \mathrm{C}$ protease [45], nucleoprotein [47] and NS5A [46].

However, our efforts lead to the identification that the C-terminal SPRY domain of TRIM22 curtailed the propagation of PRRSV, whereas the N-terminal RING domain was 
dispensable. In fact, approximately $60 \%$ of all known human TRIM also harbor a variable SPRY domain at the carboxy terminus, facilitating protein-protein interactions [62]. The TRIM22 SPRY domain was found to specifically targeting viral $\mathrm{N}$ protein, much resembling the interaction between TRIM22 and nucleoprotein of IAV [47]. As nucleocapsid protein of RNA viruses, both of them have an essential role in virus assembly, encapsidating the genomic RNA, viral replication and transcription [63]. Noteworthy, the RING domain dependent/independent restriction strategies of virus from different family by TRIM22 appears to be mechanistically similar to the antiviral effect exerted by TRIM56 against Flaviviruses, Coronavirus, and Influenza virus infection [32, 64], suggested that TRIMs have developed multiple strategy to inhibit distinct viral infection.

It is widely accepted that the antiviral activities of TRIM22 was demonstrated to coupled with the localization [41]. Of interest, regarding HIV suppression, TRIM 22 acts both in the nucleus at the level of transcription of viral DNA by blocking the action of Sp1 and in the cytoplasma at the level of assembly of new viral particles [43, 51, 65]. Moreover, Gao and colleagues observed that TRIM22 was mainly localized in the nucleus of HepG2 cells when it suppresses HBV survival [44]. In this study, it is proposed that the interaction between TRIM22 and PRRSV N protein in the nucleus might be involved in restriction of PRRSV replication.

Although PRRSV replicates in the cytoplasm, as is the case of most RNA viruses, its N protein is found in both cytoplasmic and nuclear compartments during infection [63]. Furthermore, it has been purposed that the NLS2 at position 41-47 affects $\mathrm{N}$ protein nuclear localization during viral infection and nuclear localization of the $\mathrm{N}$ protein participates in the regulation of several host cell processes [55, 66-68]. PRRSV with mutation of the NLS2 is shown to induce lower viremia, while higher neutralizing antibody level than the wild-type virus [68]. We found that $\mathrm{N}$ protein NLS2 mutants K43-2A failed to interact with TRIM22, further supporting the functional significance of this motif. Several cellular proteins have so far been demonstrated to interact with the $\mathrm{N}$ protein, as exemplified by inhibitor of MyoD family-a domain-containing protein, Poly(A)-binding protein (PABP), myxovirus resistance 2 (Mx2), DExD/HBox Helicase 9 (DHX9), DHX36, SUMO E2 conjugating enzyme Ubc9, and MOV10 [19, 49, 67, 69-72]. These interactions conferred to the regulation of PRRSV replication and immune evasion. Ongoing and future research will no doubt continue to strive to identify key interactions involved in the PRRSV-associated immune evasion.

Previous studies have shown that the pig, cow, and goat genome lose the TRIM22 gene during evolution, whereas the primates and horse genome contain TRIM22 gene [42]. Our findings provide evidence that human TRIM22 targets the N proteins from SHFV, EAV, and LDV for degradation, suggesting a broad antiviral mechanism of TRIM22 against arteriviruses. However, the expression of PRRSV N protein is resistant to TRIM22 over-expression (Fig. 5a). A possible explanation is that the $\mathrm{N}$ proteins of arteriviruses share only $\sim 40 \%$ sequence similarity of the amino acid and TRIM22 adopts distinct mechanisms towards different viral $\mathrm{N}$ proteins. In this regard, a previous report has shown that differences between the amino acid sequences of NP from several IAV strains determine the sensitivity to TRIM22mediated degradation [73].

Meanwhile, monkey kidney cell lines, including MARC145 cells, are extensively used to propagate PRRSV isolates for vaccine production [5]. Therefore, the identification that knockdown of TRIM22 in MARC-145 cells increases PRRSV titer might have promising implications in reducing vaccine production costs. Recently, the CRISPR-based technology has been applied to the generation of viral diseaseresistant pigs [74-76]. In this context, restoring expression of TRIM22 via the breeding of transgenic pigs may provide potential anti-PRRSV strategies and considerable productivity benefits to pork producers.

Acknowledgements This study was supported by Key Scientific Research Project of University in Henan Province (19A230003); the Key Technologies Research and Development Program of Henan Province (192102110176); Doctoral Science Foundation of Henan University of Animal Husbandry and Economy (53000176); Scientific Research Innovation Team of Veterinary Bio-technology (2018KYTD16); and Key Discipline of Preventive Veterinary Medicine of Henan University of Animal Husbandry and Economy (MXK2016102).

Author contributions TR and HYJ designed the study. ND, YTS, SFC, JHW, WTK, YL, and YZ performed the experiments in the study. HH and WD analyzed the data. HYJ wrote and edited the manuscript. All authors read and approved the final manuscript.

\section{Compliance with ethical standards}

Conflict of interest The authors declare that they have no competing interests.

Ethical approval This article does not contain any studies with human participants or animals performed by any of the authors.

\section{Reference}

1. Lunney JK, Fang Y, Ladinig A, Chen N, Li Y, Rowland B, Renukaradhya GJ (2016) Porcine reproductive and respiratory syndrome virus (PRRSV): pathogenesis and interaction with the immune system. Annu Rev Anim Biosci 4:129-154

2. Ke H, Yoo D (2017) The viral innate immune antagonism and an alternative vaccine design for PRRS virus. Vet Microbiol 209:75-89 
3. Pileri E, Mateu E (2016) Review on the transmission porcine reproductive and respiratory syndrome virus between pigs and farms and impact on vaccination. Vet Res 47:108

4. Popescu LN, Trible BR, Chen N, Rowland RRR (2017) Gp5 of porcine reproductive and respiratory syndrome virus (PRRSV) as a target for homologous and broadly neutralizing antibodies. Vet Microbiol 209:90-96

5. Renukaradhya GJ, Meng XJ, Calvert JG, Roof M, Lager KM (2015) Live porcine reproductive and respiratory syndrome virus vaccines: current status and future direction. Vaccine 33:4069-4080

6. Qi P, Liu K, Wei J, Li Y, Li B, Shao D, Wu Z, Shi Y, Tong G, Qiu Y, Ma Z (2017) Nonstructural protein 4 of porcine reproductive and respiratory syndrome virus modulates cell surface swine leukocyte antigen class I expression by downregulating beta2microglobulin transcription. J Virol 91:e01755

7. Yang L, Wang R, Ma Z, Xiao Y, Nan Y, Wang Y, Lin S, Zhang YJ (2017) Porcine reproductive and respiratory syndrome virus antagonizes JAK/STAT3 signaling via nsp5, which induces STAT3 degradation. J Virol 91:e02087

8. Wang R, Zhang YJ (2014) Antagonizing interferon-mediated immune response by porcine reproductive and respiratory syndrome virus. Biomed Res Int 2014:315470

9. Beura LK, Sarkar SN, Kwon B, Subramaniam S, Jones C, Pattnaik AK, Osorio FA (2010) Porcine reproductive and respiratory syndrome virus nonstructural protein 1beta modulates host innate immune response by antagonizing IRF3 activation. J Virol $84: 1574-1584$

10. Huang C, Zhang Q, Guo XK, Yu ZB, Xu AT, Tang J, Feng WH (2014) Porcine reproductive and respiratory syndrome virus nonstructural protein 4 antagonizes beta interferon expression by targeting the NF- $\mathrm{KB}$ essential modulator. J Virol 88:10934-10945

11. Wang R, Nan Y, Yu Y, Zhang YJ (2013) Porcine reproductive and respiratory syndrome virus nsplbeta inhibits interferon-activated JAK/STAT signal transduction by inducing karyopherin-alpha1 degradation. J Virol 87:5219-5228

12. Jing H, Fang L, Ding Z, Wang D, Hao W, Gao L, Ke W, Chen $\mathrm{H}, \mathrm{Xiao} \mathrm{S}$ (2017) Porcine reproductive and respiratory syndrome virus nsp $1 \alpha$ inhibits NF- $\kappa$ B activation by targeting the linear ubiquitin chain assembly complex. J Virol 91:e01911

13. Harding JCS, Ladinig A, Novakovic P, Detmer SE, Wilkinson JM, Yang T, Lunney JK, Plastow GS (2017) Novel insights into host responses and reproductive pathophysiology of porcine reproductive and respiratory syndrome caused by PRRSV-2. Vet Microbiol 209:114-123

14. Rahe MC, Murtaugh MP (2017) Mechanisms of adaptive immunity to porcine reproductive and respiratory syndrome virus. Viruses 9:148

15. Loving CL, Osorio FA, Murtaugh MP, Zuckermann FA (2015) Innate and adaptive immunity against porcine reproductive and respiratory syndrome virus. Vet Immunol Immunopathol 167:1-14

16. Snijder EJ, Kikkert M, Fang Y (2013) Arterivirus molecular biology and pathogenesis. J Gen Virol 94:2141-2163

17. Sun Y, Han M, Kim C, Calvert JG, Yoo D (2012) Interplay between interferon-mediated innate immunity and porcine reproductive and respiratory syndrome virus. Viruses 4:424-446

18. Zhang R, Chen C, Sun Z, Tan F, Zhuang J, Tian D, Tong G, Yuan S (2012) Disulfide linkages mediating nucleocapsid protein dimerization are not required for porcine arterivirus infectivity. J Virol 86:4670-4681

19. Liu L, Tian J, Nan H, Tian MM, Li Y, Xu XD, Huang BC, Zhou EM, Hiscox JA, Chen HY (2016) Porcine reproductive and respiratory syndrome virus nucleocapsid protein interacts with Nsp9 and cellular DHX9 to regulate viral RNA synthesis. J Virol 90:5384-5398
20. Song T, Fang L, Wang D, Zhang R, Zeng S, An K, Chen H, Xiao S (2016) Quantitative interactome reveals that porcine reproductive and respiratory syndrome virus nonstructural protein 2 forms a complex with viral nucleocapsid protein and cellular vimentin. J Proteomics 142:70-81

21. Liu X, Fan B, Bai J, Wang H, Li Y, Jiang P (2015) The n-n noncovalent domain of the nucleocapsid protein of type 2 porcine reproductive and respiratory syndrome virus enhances induction of IL-10 expression. J Gen Virol 96:1276-1286

22. Chen X, Zhang Q, Bai J, Zhao Y, Wang X, Wang H, Jiang $P(2017)$ The nucleocapsid protein and nonstructural protein 10 of highly pathogenic porcine reproductive and respiratory syndrome virus enhance cd83 production via NF- $\kappa B$ and $\mathrm{Sp} 1$ signaling pathways. J Virol 91:e00986

23. Fu Y, Quan R, Zhang H, Hou J, Tang J, Feng WH (2012) Porcine reproductive and respiratory syndrome virus induces interleukin-15 through the NF- $\kappa \mathrm{B}$ signaling pathway. J Virol 86:7625-7636

24. Wongyanin P, Buranapraditkul S, Yoo D, Thanawongnuwech R, Roth JA, Suradhat S (2012) Role of porcine reproductive and respiratory syndrome virus nucleocapsid protein in induction of interleukin-10 and regulatory t-lymphocytes (Treg). J Gen Virol 93:1236-1246

25. Jing H, Fang L, Wang D, Ding Z, Luo R, Chen H, Xiao S (2014) Porcine reproductive and respiratory syndrome virus infection activates NOD2-RIP2 signal pathway in MARC-145 cells. Virology 458-459:162-171

26. Fan B, Liu X, Bai J, Li Y, Zhang Q, Jiang P (2015) The $15 \mathrm{~N}$ and $46 \mathrm{R}$ residues of highly pathogenic porcine reproductive and respiratory syndrome virus nucleocapsid protein enhance regulatory T lymphocytes proliferation. PLoS ONE 10:e0138772

27. Yu J, Liu Y, Zhang Y, Zhu X, Ren S, Guo L, Liu X, Sun W, Chen Z, Cong X, Chen L, Shi J, Du Y, Li J, Wu J, Wang J (2017) The integrity of PRRSV nucleocapsid protein is necessary for up-regulation of optimal interleukin-10 through NF- $\mathrm{KB}$ and p38 MAPK pathways in porcine alveolar macrophages. Microb Pathog 109:319-324

28. Esposito D, Koliopoulos MG, Rittinger K (2017) Structural determinants of trim protein function. Biochem Soc Trans 45:183-191

29. Gushchina LV, Kwiatkowski TA, Bhattacharya S, Weisleder NL (2018) Conserved structural and functional aspects of the tripartite motif gene family point towards therapeutic applications in multiple diseases. Pharmacol Ther 185:12-25

30. Hatakeyama $S$ (2017) Trim family proteins: roles in autophagy, immunity, and carcinogenesis. Trends Biochem Sci 42:297-311

31. Kimura T, Jain A, Choi SW, Mandell MA, Johansen T, Deretic V (2017) Trim-directed selective autophagy regulates immune activation. Autophagy 13:989-990

32. Liu B, Li NL, Wang J, Shi PY, Wang T, Miller MA, Li K (2014) Overlapping and distinct molecular determinants dictating the antiviral activities of TRIM56 against flaviviruses and coronavirus. J Virol 88:13821-13835

33. Obad S, Olofsson T, Mechti N, Gullberg U, Drott K (2007) Regulation of the interferon-inducible p53 target gene TRIM22 (Staf50) in human T lymphocyte activation. J Interferon Cytokine Res 27:857-864

34. Yu S, Gao B, Duan Z, Xu W, Xiong S (2011) Identification of tripartite motif-containing 22 (TRIM22) as a novel NF- $\mathrm{kB}$ activator. Biochem Biophys Res Commun 410:247-251

35. Qiu H, Huang F, Xiao H, Sun B, Yang R (2013) Trim22 inhibits the TRAF6-stimulated NF- $\mathrm{BB}$ pathway by targeting TAB2 for degradation. Virol Sin 28:209-215

36. Chen C, Zhao D, Fang S, Chen Q, Cheng B, Fang X, Shu Q (2017) TRIM22-mediated apoptosis is associated with BAK oligomerization in monocytes. Sci Rep 7:39961 
37. Li Q, Lee CH, Peters LA, Mastropaolo LA, Thoeni C, Elkadri A, Schwerd T, Zhu J, Zhang B, Zhao Y, Hao K, Dinarzo A, Hoffman G, Kidd BA, Murchie R, Al Adham Z, Guo C, Kotlarz D, Cutz E, Walters TD, Shouval DS, Curran M, Dobrin R, Brodmerkel C, Snapper SB, Klein C, Brumell JH, Hu M, Nanan R, Snanter-Nanan B, Wong M, Le Deist F, Haddad E, Roifman CM, Deslandres C, Griffiths AM, Gaskin KJ, Uhlig HH, Schadt EE, Muise AM (2016) Variants in TRIM22 that affect NOD2 signaling are associated with very-early-onset inflammatory bowel disease. Gastroenterology 150:1196-1207

38. Lou J, Wang Y, Zheng X, Qiu W (2018) TRIM22 regulates macrophage autophagy and enhances mycobacterium tuberculosis clearance by targeting the nuclear factor-multiplicity $\mathrm{\kappa B} / \mathrm{beclin}$ 1 pathway. J Cell Biochem 119:8971-8980

39. Sivaramakrishnan G, Sun Y, Rajmohan R, Lin VC (2009) B30.2/ spry domain in tripartite motif-containing 22 is essential for the formation of distinct nuclear bodies. FEBS Lett 583:2093-2099

40. Sivaramakrishnan G, Sun Y, Tan SK, Lin VC (2009) Dynamic localization of tripartite motif-containing 22 in nuclear and nucleolar bodies. Exp Cell Res 315:1521-1532

41. Lian Q, Sun B (2017) Interferons command TRIM22 to fight against viruses. Cell Mol Immunol 14:794-796

42. Sawyer SL, Emerman M, Malik HS (2007) Discordant evolution of the adjacent antiretroviral genes TRIM22 and TRIM5 in mammals. PLoS Pathog 3:e197

43. Barr SD, Smiley JR, Bushman FD (2008) The interferon response inhibits HIV particle production by induction of trim22. PLoS Pathog 4:e1000007

44. Gao B, Duan Z, Xu W, Xiong S (2009) Tripartite motif-containing 22 inhibits the activity of hepatitis b virus core promoter, which is dependent on nuclear-located ring domain. Hepatology 50:424-433

45. Eldin P, Papon L, Oteiza A, Brocchi E, Lawson TG, Mechti N (2009) Trim 22 e3 ubiquitin ligase activity is required to mediate antiviral activity against encephalomyocarditis virus. J Gen Virol 90:536-545

46. Yang C, Zhao X, Sun D, Yang L, Chong C, Pan Y, Chi X, Gao Y, Wang M, Shi X, Sun H, Lv J, Gao Y, Zhong J, Niu J, Sun B (2016) Interferon alpha (ifnalpha)-induced TRIM22 interrupts HCV replication by ubiquitinating NS5A. Cell Mol Immunol 13:94-102

47. Di Pietro A, Kajaste-Rudnitski A, Oteiza A, Nicora L, Towers GJ, Mechti N, Vicenzi E (2013) TRIM22 inhibits influenza a virus infection by targeting the viral nucleoprotein for degradation. $\mathbf{J}$ Virol 87:4523-4533

48. Cao SF, Guo QY, Wang Y (2015) Inhibition of highly pathogenic porcine reproductive and respiratory syndrome virus replication by recombinant pseudorabies virus-mediated RNA interference in piglets. Vet Microbiol 181:212-220

49. Jing H, Zhou Y, Fang L, Ding Z, Wang D, Ke W, Chen H, Xiao S (2017) Dexd/h-box helicase 36 signaling via myeloid differentiation primary response gene 88 contributes to NF- $\mathrm{kB}$ activation to type 2 porcine reproductive and respiratory syndrome virus infection. Front Immunol 8:1365

50. Ke W, Fang L, Jing H, Tao R, Wang T, Li Y, Long S, Wang D, Xiao S (2017) Cholesterol 25-hydroxylase inhibits porcine reproductive and respiratory syndrome virus replication through enzyme activity-dependent and -independent mechanisms. J Virol 91:e00827

51. Vicenzi E, Poli G (2018) The interferon-stimulated gene TRIM22: a double-edged sword in HIV-1 infection. Cytokine Growth Factor Rev 40:40-47

52. Kajaste-Rudnitski A, Marelli SS, Pultrone C, Pertel T, Uchil PD, Mechti N, Mothes W, Poli G, Luban J, Vicenzi E (2011) Trim22 inhibits HIV-1 transcription independently of its e3 ubiquitin ligase activity, tat, and NF- $\mathrm{KB}$-responsive long terminal repeat elements. J Virol 85:5183-5196
53. Tissot C, Mechti N (1995) Molecular cloning of a new interferon-induced factor that represses human immunodeficiency virus type 1 long terminal repeat expression. J Biol Chem 270:14891-14898

54. Daginakatte GC, Kapil S (2001) Mapping of the rna-binding domain of the porcine reproductive and respiratory syndrome virus nucleocapsid protein. Adv Exp Med Biol 494:547-552

55. Rowland RR, Schneider P, Fang Y, Wootton S, Yoo D, Benfield DA (2003) Peptide domains involved in the localization of the porcine reproductive and respiratory syndrome virus nucleocapsid protein to the nucleolus. Virology 316:135-145

56. Kenney SP, Meng XJ (2015) An SH3 binding motif within the nucleocapsid protein of porcine reproductive and respiratory syndrome virus interacts with the host cellular signaling proteins STAMI, TXK, Fyn, Hck, and cortactin. Virus Res 204:31-39

57. Wootton SK, Yoo D (2003) Homo-oligomerization of the porcine reproductive and respiratory syndrome virus nucleocapsid protein and the role of disulfide linkages. J Virol 77:4546-4557

58. Singh R, Patel V, Mureithi MW, Naranbhai V, Ramsuran D, Tulsi S, Hiramen K, Werner L, Mlisana K, Altfeld M, Luban J, Kasprowicz V, Dheda K, Abdool Karim SS, Ndung'u T (2014) TRIM5 $\alpha$ and TRIM22 are differentially regulated according to HIV-1 infection phase and compartment. J Virol 88:4291-4303

59. Gao B, Xu W, Wang Y, Zhong L, Xiong S (2013) Induction of TRIM22 by IFN- $\gamma$ involves JAK and PC-PLC/PKC, but not mapks and pi3K/Akt/mTOR pathways. J Interferon Cytokine Res 33:578-587

60. Oteiza A, Mechti N (2015) Control of foxo4 activity and cell survival by TRIM22 directs TLR3-stimulated cells toward IFN type I gene induction or apoptosis. J Interferon Cytokine Res 35:859-874

61. Qiu H, Huang F, Gong J, Xiao H, Sun BL, Yang RG (2015) TRIM22 can activate the noncanonical NF- $\kappa$ B pathway by affecting ikkalpha. J Recept Signal Transduct Res 35:289-294

62. van Tol S, Hage A, Giraldo MI, Bharaj P, Rajsbaum R (2017) The trimendous role of trims in virus-host interactions. Vaccines 5:23

63. Wulan WN, Heydet D, Walker EJ, Gahan ME, Ghildyal R (2015) Nucleocytoplasmic transport of nucleocapsid proteins of enveloped rna viruses. Front Microbiol 6:553

64. Liu B, Li NL, Shen Y, Bao X, Fabrizio T, Elbahesh H, Webby RJ, Li K (2016) The c-terminal tail of TRIM56 dictates antiviral restriction of influenza a and $b$ viruses by impeding viral RNA synthesis. J Virol 90:4369-4382

65. Turrini F, Marelli S, Kajaste-Rudnitski A, Lusic M, Van Lint C, Das AT, Harwig A, Berkhout B, Vicenzi E (2015) HIV-1 transcriptional silencing caused by TRIM22 inhibition of Sp1 binding to the viral promoter. Retrovirology 12:104

66. Yoo D, Wootton SK, Li G, Song C, Rowland RR (2003) Colocalization and interaction of the porcine arterivirus nucleocapsid protein with the small nucleolar rna-associated protein fibrillarin. J Virol 77:12173-12183

67. Song C, Lu R, Bienzle D, Liu HC, Yoo D (2009) Interaction of the porcine reproductive and respiratory syndrome virus nucleocapsid protein with the inhibitor of myod family-a domain-containing protein. Biol Chem 390:215-223

68. Lee C, Hodgins D, Calvert JG, Welch SK, Jolie R, Yoo D (2006) Mutations within the nuclear localization signal of the porcine reproductive and respiratory syndrome virus nucleocapsid protein attenuate virus replication. Virology 346:238-250

69. Wang X, Bai J, Zhang L, Wang X, Li Y, Jiang P (2012) Poly(a)binding protein interacts with the nucleocapsid protein of porcine reproductive and respiratory syndrome virus and participates in viral replication. Antiviral Res 96:315-323

70. Wang H, Bai J, Fan B, Li Y, Zhang Q, Jiang P (2016) The interferon-induced $\mathrm{Mx} 2$ inhibits porcine reproductive and respiratory syndrome virus replication. J Interferon Cytokine Res 36:129-139 
71. Wang C, Zeng N, Liu S, Miao Q, Zhou L, Ge X, Han J, Guo X, Yang $H$ (2017) Interaction of porcine reproductive and respiratory syndrome virus proteins with sumo-conjugating enzyme reveals the sumoylation of nucleocapsid protein. PLoS ONE 12:e0189191

72. Zhao K, Li LW, Zhang YJ, Jiang YF, Gao F, Li GX, Yu LX, Zhao WY, Shan TL, Zhou YJ, Tong GZ (2018) Mov10 inhibits replication of porcine reproductive and respiratory syndrome virus by retaining viral nucleocapsid protein in the cytoplasm of MARC145 cells. Biochem Biophys Res Commun 504:157-163

73. Pagani I, Di Pietro A, Oteiza A, Ghitti M, Mechti N, Naffakh N, Vicenzi E (2018) Mutations conferring increased sensitivity to tripartite motif 22 restriction accumulated progressively in the nucleoprotein of seasonal influenza a $(\mathrm{H} 1 \mathrm{~N} 1)$ viruses between 1918 and 2009. mSphere 3:e00110

74. Whitworth KM, Rowland RRR, Petrovan V, Sheahan M, CinoOzuna AG, Fang Y, Hesse R, Mileham A, Samuel MS, Wells KD, Prather RS (2019) Resistance to coronavirus infection in amino peptidase n-deficient pigs. Transgenic Res 28:21-32
75. Huang G, Liu X, Tang X, Du L, Feng W, Hu X, Zhu L, Li Q, Suo $X$ (2017) Increased neutralizing antibody production and interferon-gamma secretion in response to porcine reproductive and respiratory syndrome virus immunization in genetically modified pigs. Front Immunol 8:1110

76. Burkard C, Lillico SG, Reid E, Jackson B, Mileham AJ, Ait-Ali T, Whitelaw CB, Archibald AL (2017) Precision engineering for PRRSV resistance in pigs: Macrophages from genome edited pigs lacking CD163 SRCR5 domain are fully resistant to both prrsv genotypes while maintaining biological function. PLoS Pathog 13:e1006206

Publisher's Note Springer Nature remains neutral with regard to jurisdictional claims in published maps and institutional affiliations. 